\title{
A side-sensitive synthetic chart combined with a VSS $\bar{X}$ chart
}

\author{
Antonio F.B. Costa*, Marcela A.G. Machado \\ Departamento de Produção, Faculdade de Engenharia, UNESP, Guaratinguetá, SP 12516-410, Brazil
}

\section{A R T I C L E I N F O}

\section{Article history:}

Received 3 December 2014

Received in revised form 18 September 2015

Accepted 16 October 2015

Available online 26 October 2015

\section{Keywords:}

Quality control

$\bar{X}$ chart

Synthetic

Side-sensitive

VSS

\begin{abstract}
A B S T R A C T
In independent investigations, the variable sample size (VSS) scheme and the side-sensitive synthetic (SS) rule proved to reduce the delay with which the $\bar{X}$ chart signals. Based on these findings we investigate the $\bar{X}$ chart's performance with the joint use of the VSS scheme and the side-sensitive synthetic rule. The SSVSS $\bar{X}$ chart outperforms the pure VSS $\bar{X}$ chart, especially when the risk of false alarms and the rate of inspected items per sampling cannot be high. In these cases, the VSS $\bar{X}$ chart detects moderate mean shifts (around one standard deviation) in half of the time thanks to the side-sensitive synthetic rule.
\end{abstract}

(c) 2015 Elsevier Ltd. All rights reserved.

\section{Introduction}

In 1920s, Walter A. Shewhart introduced the $\bar{X}$ control chart with the well-known signaling rule that signals when a sample point falls in the action region. The $\bar{X}$ chart with this easy-to-use rule works properly when shifts in the process mean are larger than one standard deviation - taking samples of size four, the $\bar{X}$ chart requires less than 6.3 samples to signal a shift larger than one standard deviation. For smaller shifts, the practitioners are advised to use the Shewhart charts with supplementary run rules. Champ and Woodall (1987) studied the $\bar{X}$ control chart with supplementary run rules and they concluded that with these rules the Shewhart chart is more sensitive to small shifts in the mean, but not as sensitive as the cumulative sum (CUSUM) chart. The synthetic chart proposed by Wu and Spedding (2000) is a run rules chart that employs the conforming run length $(C R L)$ to assess the state of the process. The $C R L$ is the number of conforming samples between two consecutive nonconforming samples plus the ending nonconforming one. A nonconforming sample is the one with an $\bar{X}$ value larger than the upper control limit $U C L=\mu_{0}+k \sigma_{\bar{X}}$ or smaller than the lower control limit $L C L=\mu_{0}-k \sigma_{\bar{X}}$, where $\mu_{0}$ is the in-control value of the process mean, $k$ is the width of the control limits and $\sigma_{\bar{X}}$ is the standard deviation of the sample mean. The synthetic chart signals when $C R L \leq L$, where $L$ is a specified positive integer. Davis and Woodall (2002) constructed a Markov chain

\footnotetext{
* Corresponding author.

E-mail addresses: fbranco@feg.unesp.br (A.F.B. Costa), marcela@feg.unesp.br (M.A.G. Machado).
}

model to evaluate the performance of the synthetic chart in terms of the zero-state and the steady-state average run lengths (ARLs).

The interest in synthetic charts has been growing. Costa, De Magalhaes, and Epprecht (2009) evaluated the properties of a synthetic chart with two-stage testing that is able to signal changes in the process mean and in the process variance. Machado, Costa, and Rahim (2009) studied the properties of the synthetic VMAX chart which is used to control the covariance matrix of bivariate processes. Khoo, Lee, Wu, Chen, and Castagliola (2011) proposed a synthetic double sampling (DS) chart, which combines the double sampling $\bar{X}$ chart and the $C R L$ chart for monitoring the process mean. Costa and Machado (2015) extended their study by including the side-sensitive feature and the computation of the steady-state ARL. In terms of the zero-state, the synthetic DS chart outperforms the DS chart. We cannot say the same regarding to the steady-state ARL. Inspired by the works of Khoo et al. (2011) and Haridy et al. (2012), Chong, Khoo, and Castagliola (2014) proposed a synthetic double sampling chart for attributes, which combines the double sampling $n p$ chart and the CRL chart. Recently, a lot of research in SPC has focused in synthetic charts for univariate processes, including synthetic $\bar{X}$ charts, see Wu, Ou, Castagliola, and Khoo (2010), Zhang, Castagliola, Wu, and Khoo (2011), Yeong, Khoo, Wu, and Castagliola (2012), Yeong, Khoo, Lee, and Rahim (2013), Bajirao and Parasharam (2015), Guo, Wang, and Cheng (2015), Khoo, Tan, Chong, and Haridy (2015), and Rajmanya and Ghute (2014). Khoo, Wu, Castagliola, and Lee (2013), Haridy, Wu, Abhary, Castagliola, and Shamsuzzaman (2014), Lee, Khoo, and Xie (2014) and Lee and Khoo (2015) also considered the use of synthetic charts to control multivariate processes. 
Machado and Costa (2014a) studied the side-sensitive version of the synthetic chart. The side-sensitive chart signals when two points, not far from each other and on the same side of the centerline, fall beyond the warning limit. If the side does not matter, the side-sensitive chart reduces to a synthetic chart. Machado and Costa (2014b) also extended the work of Wu et al. (2010) combining the side-sensitive synthetic chart with an $\bar{X}$ chart. The results proved that the synthetic- $\bar{X}$ chart performs better when it is side-sensitive.

The variable sample size (VSS) scheme introduced by Costa (1994) has also the purpose to improve the ability of the $\bar{X}$ chart in signaling mean shifts smaller than one and a half standard deviations. According to the VSS scheme, the position of the last sample mean defines the size of the next sample. If the sample mean falls in the central region, then it is reasonable to relax the control by decreasing the size of the next sample. Otherwise, if the sample mean falls in the warning region, then it is reasonable to tighten the control by increasing the size of the next sample. The idea of varying not only the size of the samples but also the sampling intervals and/or the control limits was also explored by Costa (1997, 1998, 1999a, 1999b). Recent papers dealing with $\bar{X}$ charts with variable parameters include Castagliola, Zhang, Costa, and Maravelakis (2012), Costa and Machado (2011), Lee (2013), Chew, Khoo, Teh, Castagliola, and Industrial Engineering (2015), Lim, Khoo, Teoh, and Xie (2015) and Noorossana, Shekary, and Deheshvar (2015).

In this article, we propose two VSS $\bar{X}$ charts; the first one is combined with a synthetic chart (SVSS $\bar{X}$ chart) and the second one is combined with a side-sensitive synthetic chart (SSVSS $\bar{X}$ chart). The Markov chain approach helped to obtain the properties of the proposed charts.

\section{Assumptions and the measures of performance}

The SVSS and SSVSS $\bar{X}$ charts are employed to monitor a process whose quality characteristic of interest $(X)$ is normally distributed, $X \sim N\left(\mu ; \sigma^{2}\right)$. The process starts and remains in a state of statistical control until the occurrence of an assignable cause. During the incontrol period $\mu=\mu_{0}$ and after that the mean shifts to $\mu_{1}=\mu_{0}+\delta \sigma$. After a true alarm, the assignable cause is eliminated and the process is brought back to the state of statistical control.

According to Davis and Woodall (2002), the proper quantity to measure the performance of a synthetic chart is the steady state $A R L$ (SSARL) - the ARL under the assumption that the process remains in-control for a long time before the occurrence of the assignable cause. There are many other quantities to measure the performance of the control charts. For example, Wu, Yang, Jiang, and Khoo (2008) used the Extra Quadratic Loss (EQL) to measure and compare the performance of the charts, which is given by:

$E Q L=\frac{\sigma^{2}}{\delta_{\max }} \int_{0}^{\delta_{\max }} \delta^{2} \operatorname{SSARL}(\delta) d \delta$

where $\operatorname{SSARL}(\delta)$ is the SSARL computed with $\mu=\mu_{1}$ and $\delta_{\max }$ is the upper bound of $\delta$. Expression (1), assumes that all mean shifts within the range $\left(0<\delta \leqslant \delta_{\max }\right)$ occur with equal probability, that is, the magnitude of the shift, $\delta$, is a random variable with uniform distribution (see Wu et al. (2008) for details).

\section{The VSS $\bar{X}$ chart}

When the VSS scheme is in use, random samples with variable size, switching between small $\left(n_{1}\right)$ and large $\left(n_{2}\right)$ sizes, are taken at regular intervals of time. The standardized sample means
$Z\left(n_{i}\right)=\left(\bar{X}-\mu_{0}\right) \sqrt{n_{i}} / \sigma$ are plotted on a control chart with warning $( \pm w)$ and action $( \pm k)$ limits.

The size of each sample depends on the point position of the preceding sample. If the current $Z\left(n_{i}\right)$ point falls in the central region, then the next sample size will be $n_{1}$, and if the current $Z\left(n_{i}\right)$ point falls in the warning region, then the next sample size will be $n_{2}$. The chart produces a signal when a sample point falls in the action region. The specifications of $w$ and $k$ take into account the constraint that during the in-control period the conditional probability $p_{0}$, of a sample point falling in the central region, given that it did not fall in the action region, is:

$p_{0}=\operatorname{Pr}\left[\left|Z\left(n_{i}\right)\right|<w|| Z\left(n_{i}\right) \mid<k\right]$

The size of the first sample that is taken from the process when it is just starting, or after a false alarm, is randomly decided to be small (large) with probability $p_{0}\left(1-p_{0}\right)$. During the in-control period, all samples have a probability $p_{0}$ of being small and a probability of $1-p_{0}$ of being large.

During the in-control period, the rate of inspected items per sampling $(\bar{n})$ is given by

$\bar{n}=n_{1} p_{0}+n_{2}\left(1-p_{0}\right)$

The steady-state ARL of the VSS chart is (see Costa (1994) for details):

$$
\begin{aligned}
\text { SSARL }= & p_{0}\left[\frac{1-p_{22}+p_{12}}{\left(1-p_{11}\right)\left(1-p_{22}\right)-p_{12} p_{21}}\right] \\
& +\left(1-p_{0}\right)\left[\frac{1-p_{11}+p_{21}}{\left(1-p_{11}\right)\left(1-p_{22}\right)-p_{12} p_{21}}\right]
\end{aligned}
$$

where

$$
\begin{aligned}
& p_{11}=\operatorname{Pr}\left[\left|Z\left(n_{1}\right)\right|<w \mid \mu=\mu_{1}\right] \\
& p_{12}=\operatorname{Pr}\left[w<\left|Z\left(n_{1}\right)\right|<k \mid \mu=\mu_{1}\right] \\
& p_{21}=\operatorname{Pr}\left[\left|Z\left(n_{2}\right)\right|<w \mid \mu=\mu_{1}\right] \\
& p_{22}=\operatorname{Pr}\left[w<\left|Z\left(n_{2}\right)\right|<k \mid \mu=\mu_{1}\right]
\end{aligned}
$$

\section{The VSS synthetic charts}

\subsection{The side-sensitive synthetic $\bar{X}$ chart with variable sample size (SSVSS $\bar{X}$ chart)}

The side-sensitive synthetic chart combined with the VSS $\bar{X}$ chart (SSVSS chart) signals when a sample point falls in the action region or two points, not far from each other and on the same side of the center line, fall in the warning region-two, see Fig. 1. To measure the distance between these two points, the conforming run length $(C R L)$ is defined by taking into consideration the following stratification; samples with their $\bar{X}$ values in the warning regiontwo are nonconforming and samples with their $\bar{X}$ values in the central region or even in the warning region-one are conforming. The $C R L$ is the number of conforming samples between two consecutive nonconforming samples plus the ending nonconforming one; in other words, the first of the two consecutive nonconforming samples is the reference to compute the CRL. A CRL lower than or equal to a specified positive integer $L(C R L<L)$ triggers a signal, except for the case when the $\bar{X}$ values of the two consecutive nonconforming samples fall in different warning regions. If the $\bar{X}$ value of the first nonconforming sample falls in the (lower) upper warning region-two and $\bar{X}$ value of the second nonconforming sample falls in the (upper) lower warning region-two the proposed chart doesn't signal but, similarly to the cases where the $C R L>L$, the ending nonconforming sample becomes the reference to compute the next CRL. The construction of the transition probabilities matrix 


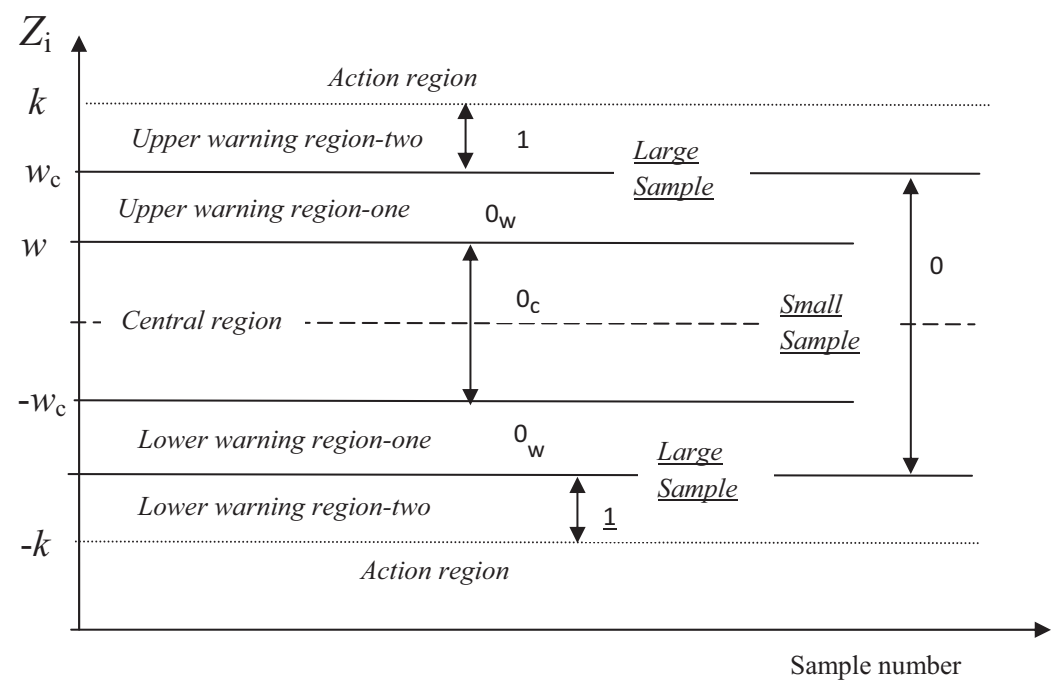

Fig. 1. The SSVSS $\bar{X}$ chart.

Table 1

The one-step transition between two states of transient matrix.

\begin{tabular}{|c|c|c|c|c|}
\hline State $(i)$ & $p_{i j}$ & State $(j)$ & $p_{i j}$ & State $(i)$ \\
\hline \multirow{5}{*}{$00 . .00 . .00_{c}$} & $\mathrm{~A}^{-}$ & $00 . .00 .00_{c}$ & $\mathrm{~A}^{+}$ & \multirow{5}{*}{$00 . .00 . .00_{w}$} \\
\hline & $\mathrm{B}^{-}$ & $00 . .00 .00_{w}$ & $\mathrm{~B}^{+}$ & \\
\hline & $\mathrm{D}^{-}$ & Signal & $\mathrm{D}^{+}$ & \\
\hline & $\mathrm{C}^{-}$ & 00..00..01 & $\mathrm{C}^{+}$ & \\
\hline & $\underline{\mathrm{C}}^{-}$ & 00..00..01 & $\underline{\mathrm{C}}^{+}$ & \\
\hline \multirow{6}{*}{$00 . .00 . .01$} & & 00..00..01 & $\mathrm{C}^{+}$ & \multirow{6}{*}{$00 . .00 . .01$} \\
\hline & $\mathrm{B}^{+}$ & $00 . .00 . .10_{w}$ & & \\
\hline & $\mathrm{A}^{+}$ & $00 . .00 . .10_{c}$ & & \\
\hline & $\mathrm{C}^{+}+\mathrm{D}^{+}$ & Signal & $\underline{\mathrm{C}}^{+}+\mathrm{D}^{+}$ & \\
\hline & & $00 . .00 . .10_{c}$ & $\overline{\mathrm{A}}^{+}$ & \\
\hline & & $00 . .00 . .10_{w}$ & $\mathrm{~B}^{+}$ & \\
\hline \multirow{6}{*}{$00 . .01 . .00_{c}$} & $\underline{\mathrm{C}}^{+}$ & 00..00..01 & & \multirow{6}{*}{$00 . .01 . .00_{w}$} \\
\hline & $\bar{A}^{-}$ & $00 . .10 . .00_{c}$ & $\mathrm{~A}^{+}$ & \\
\hline & $\mathrm{B}^{-}$ & $00 . .10 . .00_{w}$ & $\mathrm{~B}^{+}$ & \\
\hline & $\mathrm{C}^{-}+\mathrm{D}^{-}$ & Signal & $\mathrm{C}^{+}+\mathrm{D}^{+}$ & \\
\hline & $\underline{\mathrm{C}}^{-}$ & 00..00..01 & $\underline{\mathrm{C}}^{+}$ & \\
\hline & $\overline{\mathrm{A}}^{-}$ & $00 . .10 .00_{c}$ & $\bar{A}^{+}$ & \\
\hline \multirow{3}{*}{$00 . .01 . .00_{c}$} & $\mathrm{~B}^{-}$ & $00 . .10 .00_{w}$ & $\mathrm{~B}^{+}$ & \multirow{3}{*}{$00 . .01 . . .00_{w}$} \\
\hline & $\underline{\mathrm{C}}^{-}+\mathrm{D}^{-}$ & Signal & $\underline{\mathrm{C}}^{+}+\mathrm{D}^{+}$ & \\
\hline & $\mathrm{C}^{-}$ & 00..00..01 & $\mathrm{C}^{+}$ & \\
\hline
\end{tabular}

(TP matrix) is the first step to obtain the steady-state ARLs (SSARLs) of the SSVSS $\bar{X}$ chart. The general form of the TP matrix is in (5); with $L=3$, the TP matrix reduces to the one in (6).

According to Fig. 1 , the $Z\left(n_{i}\right)=\left(\bar{X}-\mu_{0}\right) \sqrt{n_{i}} / \sigma$ values, shortly $Z$ values, of the last $L$ samples, including the current sample, define the transient states: " 0 " means $|Z|<w_{c}$, " 1 " means $-k<Z<-w_{c}$, and " 1 " means $w_{c}<Z<k$. The position of the current sample, shortly $Z_{c}$, also defines the size of the next sample, because of that, when the current sample is conforming $\left(\left|Z_{c}\right|<w_{c}\right)$, it is necessary to distinguish if $\left|Z_{c}\right|<w$ (represented by " $0_{c}$ ") or $w<\left|Z_{c}\right|<w_{c}$ (represented by " $0_{w}$ "). If $\left|Z_{c}\right|<w$ the next sample will be small, but if $w<\left|Z_{c}\right|<k$ (union of " 1 ", " 1 " and " $0_{w}$ ") the next sample will be large.

The one-step transitions between two states of the transient matrix (5) are in Table 1. According to Table 1, if the current state is state $(00 \ldots 01),\left(0 \ldots 010_{c}\right), \quad\left(0 \ldots 010_{w}\right), \ldots,\left(001 \ldots 0_{c}\right)$, $\left(001 \ldots 0_{w}\right),\left(010 \ldots 0_{c}\right),\left(010 \ldots 0_{w}\right),\left(100 \ldots 0_{c}\right)$ or $\left(100 \ldots 0_{w}\right)$ and the next sample point falls in the lower warning region-two, $-k<Z<-w_{c}$, the Markov chain moves to state $00 \ldots 01$.
Similarly, if the current state is state $(00 \ldots 0 \underline{1}),\left(0 \ldots 010_{c}\right)$, $\left(0 \ldots 010_{w}\right), \ldots,\left(001 \ldots 0_{c}\right),\left(00 \underline{1} \ldots 0_{w}\right),\left(0 \underline{10} \ldots 0_{c}\right),\left(0 \underline{10} \ldots 0_{w}\right)$, $\left(100 \ldots 0_{c}\right)$ or $\left(100 \ldots 0_{w}\right)$ and the next sample point falls in the upper warning region-two, $w_{c}<Z<k$, the Markov chain moves to state $00 . .01$.

The approach based on the TP matrix to study the performance of the side-sensitive synthetic $\bar{X}$ chart (SS chart) can also be used to obtain the SSARLs of the $\bar{X}$ chart with the supplementary run rules suggested by the Western Electrical Handbook (1956), shortly the RR chart. The difference lies on the set of transient states. For instance, the side-sensitive $\bar{X}$ chart with $L=2$ requires five transient states: (00), (01), (01 1$),(10),(10)$, whereas the $\bar{X}$ chart with the two-of-three supplementary rule requires these same five transient states plus (11) and (11). Khoo and Ariffin (2006), Acosta-Mejia (2007) and Antzoulakos and Rakitzis (2008) discussed the Shewhart $\bar{X}$ charts and their delay reduction in signaling small shifts thanks to the use of the supplementary run rules. One of the Reviewers proved that the side-sensitive rule is exactly the revised $2-$ of $-L+1$ rule proposed by Antzoulakos and Rakitzis (2008). 


\begin{tabular}{|c|c|c|c|c|c|c|c|c|c|c|c|c|c|c|c|c|c|c|c|}
\hline & $\begin{array}{c}\underline{1} \\
0 \\
\ldots \\
0 \\
0_{w}\end{array}$ & $\begin{array}{c}\underline{1} \\
0 \\
\ldots \\
0 \\
0_{c}\end{array}$ & $\begin{array}{c}0 \\
\underline{1} \\
\ldots \\
0 \\
0_{w}\end{array}$ & $\begin{array}{l}0 \\
\underline{1} \\
\ldots \\
0 \\
0_{c}\end{array}$ & $\cdots$ & $\begin{array}{c}0 \\
0 \\
\ldots \\
\underline{1} \\
0_{\mathrm{w}}\end{array}$ & $\begin{array}{c}0 \\
0 \\
\ldots \\
\underline{1} \\
0_{c}\end{array}$ & $\begin{array}{c}0 \\
0 \\
\ldots \\
0 \\
1\end{array}$ & $\begin{array}{c}0 \\
0 \\
\ldots \\
0 \\
0_{w}\end{array}$ & $\begin{array}{c}0 \\
0 \\
\ldots \\
0 \\
0_{c}\end{array}$ & $\begin{array}{c}0 \\
0 \\
\ldots \\
0 \\
1\end{array}$ & $\begin{array}{c}0 \\
0 \\
\ldots \\
1 \\
0_{w}\end{array}$ & $\begin{array}{c}0 \\
0 \\
\ldots \\
1 \\
0_{c}\end{array}$ & & $\begin{array}{c}0 \\
1 \\
\ldots \\
0 \\
0_{w}\end{array}$ & $\begin{array}{c}0 \\
1 \\
\ldots \\
0 \\
0_{c}\end{array}$ & $\begin{array}{c}1 \\
0 \\
\ldots \\
0 \\
0_{w}\end{array}$ & $\begin{array}{c}1 \\
0 \\
\ldots \\
0 \\
0_{c}\end{array}$ & Signal \\
\hline $10 \ldots 00_{\mathrm{w}}$ & 0 & 0 & 0 & 0 & & 0 & 0 & 0 & $\mathrm{~B}^{+}$ & $\mathrm{A}^{+}$ & $\mathrm{C}^{+}$ & 0 & 0 & $\cdots$ & 0 & 0 & 0 & 0 & $\mathrm{D}^{+}+\mathrm{C}^{+}$ \\
\hline $10 \ldots 00_{\mathrm{c}}$ & 0 & 0 & 0 & 0 & 4 & 0 & 0 & 0 & $\mathrm{~B}^{-}$ & $\mathbf{A}^{-}$ & $\mathrm{C}^{-}$ & 0 & 0 & $\cdots$ & 0 & 0 & 0 & 0 & $\mathbf{D}^{-}+{\underline{\underline{C^{-}}}}^{-}$ \\
\hline $01 \ldots 00_{w}$ & $\mathbf{B}^{+}$ & $\mathbf{A}^{+}$ & 0 & 0 & & 0 & 0 & 0 & 0 & 0 & $\mathrm{C}^{+}$ & 0 & 0 & $\cdots$ & 0 & 0 & 0 & 0 & $\mathbf{D}^{+}+\underline{\mathbf{C}}^{+}$ \\
\hline $0 \underline{1} \ldots 00_{\mathrm{c}}$ & $\mathrm{B}^{-}$ & $\mathrm{A}^{-}$ & 0 & 0 & . & 0 & 0 & 0 & 0 & 0 & $\mathrm{C}^{-}$ & 0 & 0 & $\cdots$ & 0 & 0 & 0 & 0 & $\mathrm{D}^{-}+\mathrm{C}^{-}$ \\
\hline$\ldots$ & $\cdots$ & $\cdots$ & $\cdots$ & $\cdots$ & $\cdots$ & $\ldots$ & $\cdots$ & $\cdots$ & $\ldots$ & $\cdots$ & $\ldots$ & $\cdots$ & $\cdots$ & $\ldots$ & $\cdots$ & $\cdots$ & $\cdots$ & $\cdots$ & $\ldots$ \\
\hline $00 \ldots 10_{w}$ & 0 & 0 & $\mathbf{B}^{+}$ & $\mathbf{A}^{+}$ & & 0 & 0 & 0 & 0 & 0 & $\mathrm{C}^{+}$ & 0 & 0 & $\cdots$ & 0 & 0 & 0 & 0 & $\mathbf{D}^{+}+\underline{\mathbf{C}}^{+}$ \\
\hline $00 \ldots 10_{c}$ & 0 & 0 & $\mathrm{~B}^{-}$ & $\mathbf{A}^{-}$ & $\cdots$ & 0 & 0 & 0 & 0 & 0 & $\mathrm{C}^{-}$ & 0 & 0 & $\cdots$ & 0 & 0 & 0 & 0 & $\mathrm{D}^{-}+\underline{\mathrm{C}}^{-}$ \\
\hline $00 \ldots 0 \underline{1}$ & 0 & 0 & 0 & 0 & & $\mathrm{~B}^{+}$ & $\mathbf{A}^{+}$ & 0 & 0 & 0 & $\mathrm{C}^{+}$ & 0 & 0 & $\cdots$ & 0 & 0 & 0 & 0 & $\mathbf{D}^{+}+\underline{C}^{+}$ \\
\hline $00 \ldots 00_{w}$ & 0 & 0 & 0 & 0 & 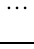 & 0 & 0 & $\underline{\mathrm{C}^{-}}$ & $\mathrm{B}^{-}$ & $\mathbf{A}^{-}$ & $\mathrm{C}^{-}$ & 0 & 0 & $\cdots$ & 0 & 0 & 0 & 0 & $\mathbf{D}^{+}$ \\
\hline $00 \ldots 00_{c}$ & 0 & 0 & 0 & 0 & & 0 & 0 & $\underline{\mathbf{C}}^{+}$ & $\mathbf{B}^{+}$ & $\mathbf{A}^{+}$ & $\mathrm{C}^{+}$ & 0 & 0 & $\cdots$ & 0 & 0 & 0 & 0 & $\mathbf{D}^{-}$ \\
\hline $00 \ldots 01$ & 0 & 0 & 0 & 0 & & 0 & 0 & $\underline{C}^{+}$ & 0 & 0 & 0 & $\mathbf{A}^{+}$ & $\mathrm{B}^{+}$ & $\mid \cdots$ & 0 & 0 & 0 & 0 & $\mathbf{D}^{+}+\mathbf{C}^{+}$ \\
\hline $00 \ldots 10_{w}$ & 0 & 0 & 0 & 0 & & 0 & 0 & $\mathrm{C}^{-}$ & 0 & 0 & 0 & 0 & 0 & $\cdots$ & $\mathbf{A}^{-}$ & $\mathrm{B}^{-}$ & 0 & 0 & $\mathrm{D}^{-}+\mathrm{C}^{-}$ \\
\hline $00 \ldots 10_{c}$ & 0 & 0 & 0 & 0 & & 0 & 0 & $\underline{\mathbf{C}}^{+}$ & 0 & 0 & 0 & 0 & 0 & ‥ & $\mathbf{A}^{+}$ & $\mathbf{B}^{+}$ & 0 & 0 & $\mathbf{D}^{+}+\mathbf{C}^{+}$ \\
\hline$\cdots$ & $\cdots$ & $\cdots$ & $\cdots$ & $\cdots$ & $\cdots$ & $\cdots$ & $\cdots$ & $\cdots$ & $\cdots$ & $\cdots$ & $\cdots$ & $\cdots$ & $\cdots$ & $\cdots$ & $\cdots$ & $\cdots$ & $\cdots$ & $\cdots$ & $\cdots$ \\
\hline $01 \ldots 00_{w}$ & 0 & 0 & 0 & 0 & & 0 & 0 & $\mathrm{C}^{-}$ & 0 & 0 & 0 & 0 & 0 & & 0 & 0 & $\mathbf{A}^{-}$ & $\mathrm{B}^{-}$ & $\mathbf{D}^{-}+\mathbf{C}^{-}$ \\
\hline $01 \ldots 00_{c}$ & 0 & 0 & 0 & 0 & & 0 & 0 & $\underline{\underline{C}}^{+}$ & 0 & 0 & 0 & 0 & 0 & & 0 & 0 & $\mathrm{~A}^{+}$ & $\mathrm{B}^{+}$ & $\mathbf{D}^{+}+\mathbf{C}^{+}$ \\
\hline $10 \ldots 00_{\mathrm{w}}$ & 0 & 0 & 0 & 0 & & 0 & 0 & $\underline{C}^{-}$ & $\mathrm{B}^{-}$ & $\mathbf{A}^{-}$ & 0 & 0 & 0 & & 0 & 0 & 0 & 0 & $\mathbf{D}^{-}+\mathrm{C}^{-}$ \\
\hline $10 \ldots 00_{\mathrm{c}}$ & 0 & 0 & 0 & 0 & & 0 & 0 & $\mathrm{C}^{+}$ & $\mathbf{B}^{+}$ & $\mathbf{A}^{+}$ & 0 & 0 & 0 & & 0 & 0 & 0 & 0 & $\mathbf{D}^{+}+\mathrm{C}^{+}$ \\
\hline Signal & 0 & 0 & 0 & 0 & & 0 & 0 & 0 & 0 & 0 & 0 & 0 & 0 & $\cdots$ & 0 & 0 & 0 & 0 & 1 \\
\hline
\end{tabular}

\begin{tabular}{|c|c|c|c|c|c|c|c|c|c|c|c|c|c|}
\hline & $\begin{array}{c}\underline{1} \\
0 \\
0_{w}\end{array}$ & $\begin{array}{l}\underline{1} \\
0 \\
0_{c}\end{array}$ & $\begin{array}{c}0 \\
\underline{1} \\
0_{\mathrm{w}}\end{array}$ & $\begin{array}{l}0 \\
\underline{1} \\
0_{c}\end{array}$ & $\begin{array}{l}0 \\
0 \\
1\end{array}$ & $\begin{array}{c}0 \\
0 \\
0_{\mathrm{w}}\end{array}$ & $\begin{array}{c}0 \\
0 \\
0_{c}\end{array}$ & $\begin{array}{l}0 \\
0 \\
1\end{array}$ & $\begin{array}{c}0 \\
1 \\
0_{w}\end{array}$ & $\begin{array}{l}0 \\
1 \\
0_{c}\end{array}$ & $\begin{array}{c}1 \\
0 \\
0_{w}\end{array}$ & $\begin{array}{c}1 \\
0 \\
0_{c}\end{array}$ & Signal \\
\hline $100_{\mathrm{w}}$ & 0 & 0 & 0 & 0 & 0 & $\mathrm{~B}^{+}$ & $\mathbf{A}^{+}$ & $\mathrm{C}^{+}$ & 0 & 0 & 0 & 0 & $\mathbf{D}^{+}+\mathbf{C}^{+}$ \\
\hline $100_{\mathrm{c}}$ & 0 & 0 & 0 & 0 & 0 & $\mathrm{~B}^{-}$ & $\mathrm{A}^{-}$ & $\mathrm{C}^{-}$ & 0 & 0 & 0 & 0 & $\mathbf{D}^{-}+\underline{\underline{C}}^{-}$ \\
\hline $010_{\mathrm{w}}$ & $\mathrm{B}^{+}$ & $\mathbf{A}^{+}$ & 0 & 0 & 0 & 0 & 0 & $\mathrm{C}^{+}$ & 0 & 0 & 0 & 0 & $\mathbf{D}^{+}+\mathbf{C}^{+}$ \\
\hline $010_{c}$ & $\mathrm{~B}^{-}$ & $\mathrm{A}^{-}$ & 0 & 0 & 0 & 0 & 0 & $\mathrm{C}^{-}$ & 0 & 0 & 0 & 0 & $\mathrm{D}^{-}+\mathrm{C}^{-}$ \\
\hline 001 & 0 & 0 & $\mathbf{B}^{+}$ & $\mathbf{A}^{+}$ & 0 & 0 & 0 & $\mathrm{C}^{+}$ & 0 & 0 & 0 & 0 & $\mathbf{D}^{+}+\underline{\mathbf{C}}^{+}$ \\
\hline $000_{\mathrm{w}}$ & 0 & 0 & 0 & 0 & $\underline{C}^{-}$ & $\mathrm{B}^{-}$ & $\mathrm{A}^{-}$ & $\mathrm{C}^{-}$ & 0 & 0 & 0 & 0 & $\mathrm{D}^{+}$ \\
\hline $000_{\mathrm{c}}$ & 0 & 0 & 0 & 0 & $\underline{C}^{+}$ & $\mathbf{B}^{+}$ & $\mathbf{A}^{+}$ & $\mathrm{C}^{+}$ & 0 & 0 & 0 & 0 & $\mathrm{D}^{-}$ \\
\hline 001 & 0 & 0 & 0 & 0 & $\underline{C}^{+}$ & 0 & 0 & 0 & $\mathbf{A}^{+}$ & $\mathrm{B}^{+}$ & 0 & 0 & $\mathrm{D}^{+}+\mathrm{C}^{+}$ \\
\hline $010_{\mathrm{w}}$ & 0 & 0 & 0 & 0 & $\underline{\mathbf{C}^{-}}$ & 0 & 0 & 0 & 0 & 0 & $\mathbf{A}^{-}$ & $\mathrm{B}^{-}$ & $\mathbf{D}^{-}+\mathbf{C}^{-}$ \\
\hline $010_{\mathrm{c}}$ & 0 & 0 & 0 & 0 & $\underline{\mathrm{C}}^{+}$ & 0 & 0 & 0 & 0 & 0 & $\mathbf{A}^{+}$ & $\mathbf{B}^{+}$ & $\mathbf{D}^{+}+\mathrm{C}^{+}$ \\
\hline $100_{\mathrm{w}}$ & 0 & 0 & 0 & 0 & $\underline{\mathbf{C}}^{-}$ & B $^{-}$ & $\mathbf{A}^{-}$ & 0 & 0 & 0 & 0 & 0 & $\mathbf{D}^{-}+\mathbf{C}^{-}$ \\
\hline $100_{\mathrm{c}}$ & 0 & 0 & 0 & 0 & $\underline{C}^{+}$ & $\mathrm{B}^{+}$ & $\mathbf{A}^{+}$ & 0 & 0 & 0 & 0 & 0 & $\mathrm{D}^{+}+\mathrm{C}^{+}$ \\
\hline Signal & 0 & 0 & 0 & 0 & 0 & 0 & 0 & 0 & 0 & 0 & 0 & 0 & 1 \\
\hline
\end{tabular}


Table 2

The probabilities of the transient matrix (5).

\begin{tabular}{|c|c|}
\hline$A^{+}=\operatorname{Pr}\left[\left|Z\left(n_{2}\right)+\delta \sqrt{n_{2}}\right|<w\right]$ & $A^{-}=\operatorname{Pr}\left[\left|Z\left(n_{1}\right)+\delta \sqrt{n_{1}}\right|<w\right]$ \\
\hline$B^{+}=\operatorname{Pr}\left[\left|Z\left(n_{2}\right)+\delta \sqrt{n_{2}}\right|<w_{c}\right]-\operatorname{Pr}\left[\left|Z\left(n_{2}\right)+\delta \sqrt{n_{2}}\right|<w\right]$ & $B^{-}=\operatorname{Pr}\left[\left|Z\left(n_{1}\right)+\delta \sqrt{n_{1}}\right|<w_{c}\right]-\operatorname{Pr}\left[\left|Z\left(n_{1}\right)+\delta \sqrt{n_{1}}\right|<w\right]$ \\
\hline$C^{+}=\operatorname{Pr}\left[w_{c}<Z\left(n_{2}\right)+\delta \sqrt{n_{2}}<k\right]$ & $C^{-}=\operatorname{Pr}\left[w_{c}<Z\left(n_{1}\right)+\delta \sqrt{n_{1}}<k\right]$ \\
\hline$\underline{C}^{+}=\operatorname{Pr}\left[-k<Z\left(n_{2}\right)+\delta \sqrt{n_{2}}<-w_{c}\right]$ & $\underline{C}^{-}=\operatorname{Pr}\left[-k<Z\left(n_{1}\right)+\delta \sqrt{n_{1}}<-w_{c}\right]$ \\
\hline $\bar{D}^{+}=\operatorname{Pr}\left[\left|Z\left(n_{2}\right)+\delta \sqrt{n_{2}}\right|>k\right]$ & $\bar{D}^{-}=\operatorname{Pr}\left[\left|Z\left(n_{1}\right)+\delta \sqrt{n_{1}}\right|>k\right]$ \\
\hline
\end{tabular}

The probabilities of the events " 1 ", " $0_{w}$ ", " $0_{c}$ " and " 1 " depend on the sample size; with large samples, they occur with probabilities $C^{+}, B^{+}, A^{+}$and $C^{+}$, and with small samples, they occur with probabilities $\underline{C}^{-}, B^{-}, A^{-}$and $C^{-}$, see Table 2. The absorbing state can be reached from any transient state. Depending on the transient state, the absorbing state is reached with probability $D^{+}, D^{-}, D^{+}+C^{+}, D^{+}+\underline{C}^{+}, D^{-}+C^{-}$, or $D^{-}+\underline{C}^{-}$. During the in-control period $\delta=0$, consequently, $A^{+}=A^{-}=A, B^{+}=B^{-}=B$, $\mathrm{C}^{+}=\mathrm{C}^{-}=\underline{C}^{+}=\underline{C}^{-}=C$, and $D^{+}=D^{-}=D$.

The SSARL is the resulting product of $\mathbf{S}^{\prime} \mathbf{A R L}$, where $\mathbf{S}$ is the vector with the stationary probabilities of being in each nonabsorbing state and ARL is the vector of ARLs taking each nonabsorbing state as the initial state. The $\mathbf{A R L}$ is given by $(\mathbf{I}-\mathbf{R})^{-1} 1$, where $\mathbf{I}$ is a $4 L \times 4 L$ identity matrix, $\mathbf{R}$ is the transition matrix given in (5) with the last row and column removed, and $\mathbf{1}$ is a $4 L \times 1$ vector of ones. The $\mathbf{S}$ vector is the solution of $\mathbf{S}^{\prime} \mathbf{R}_{\mathbf{a d j}}=\mathbf{S}$, constrained to $\mathbf{S}^{\prime} \mathbf{1}=1$. The matrix $\mathbf{R}_{\mathbf{a d j}}$ is an adjusted version of $\mathbf{R}$, where the non-zero cells of all lines are divided by $1-D-C$, except the non-zero cells of the two central lines that are divided by $1-D$. The matrix $\mathbf{R}_{\mathbf{a d j}}$ is in (7). The $S(i)$ elements of $\mathbf{S}$ are given by $g^{-1} S_{\mathbf{C}}(i)$, where $g$ is the sum of $S_{\mathbf{C}}(i), i=1,2, \ldots, 4 L$. The $4 L$ elements $S_{\mathbf{c}}(i)$ of vector $\mathbf{S}_{\mathbf{c}}$ are in Table 3, with $E=A /(1-D-C)$, $F=B /(1-D-C), \quad G=C /(1-D-C), \quad A_{0}=A /(1-D), \quad B_{0}=$ $B /(1-D)$, and $C_{0}=C /(1-D)$. The Average Number of Observations to Signal (ANOS) has also been used to compare the performance of the VSS charts. The steady state ANOS is the resulting product of $\mathbf{S}^{\prime}(\mathbf{I}-\mathbf{R})^{-1} \mathbf{N}$; the $4 L$ elements $N(i)$ of vector $\mathbf{N}$ are also in Table 3.

$\left(\begin{array}{cccccccccccccccccc}0 & 0 & 0 & 0 & \ldots & 0 & 0 & 0 & F & E & G & 0 & 0 & \ldots & 0 & 0 & 0 & 0 \\ 0 & 0 & 0 & 0 & \ldots & 0 & 0 & 0 & F & E & G & 0 & 0 & \ldots & 0 & 0 & 0 & 0 \\ F & E & 0 & 0 & \ldots & 0 & 0 & 0 & 0 & 0 & G & 0 & 0 & \ldots & 0 & 0 & 0 & 0 \\ F & E & 0 & 0 & \ldots & 0 & 0 & 0 & 0 & 0 & G & 0 & 0 & \ldots & 0 & 0 & 0 & 0 \\ \ldots & \ldots & \ldots & \ldots & \ldots & \ldots & \ldots & \ldots & \ldots & \ldots & \ldots & \ldots & \ldots & \ldots & \ldots & \ldots & \ldots & \ldots \\ 0 & 0 & F & E & \ldots & 0 & 0 & 0 & 0 & 0 & G & 0 & 0 & \ldots & 0 & 0 & 0 & 0 \\ 0 & 0 & F & E & \ldots & 0 & 0 & 0 & 0 & 0 & G & 0 & 0 & \ldots & 0 & 0 & 0 & 0 \\ 0 & 0 & 0 & 0 & \ldots & F & E & 0 & 0 & 0 & G & 0 & 0 & \ldots & 0 & 0 & 0 & 0 \\ 0 & 0 & 0 & 0 & \ldots & 0 & 0 & C_{0} & B_{0} & A_{0} & C_{0} & 0 & 0 & \ldots & 0 & 0 & 0 & 0 \\ 0 & 0 & 0 & 0 & \ldots & 0 & 0 & C_{0} & B_{0} & A_{0} & C_{0} & 0 & 0 & \ldots & 0 & 0 & 0 & 0 \\ 0 & 0 & 0 & 0 & \ldots & 0 & 0 & G & 0 & 0 & 0 & E & F & \ldots & 0 & 0 & 0 & 0 \\ 0 & 0 & 0 & 0 & \ldots & 0 & 0 & G & 0 & 0 & 0 & E & F & \ldots & 0 & 0 & 0 & 0 \\ \ldots & \ldots & \ldots & \ldots & \ldots & \ldots & \ldots & \ldots & \ldots & \ldots & \ldots & \ldots & \ldots & \ldots & \ldots & \ldots & \ldots & \ldots \\ 0 & 0 & 0 & 0 & \ldots & 0 & 0 & G & 0 & 0 & 0 & E & F & \ldots & 0 & 0 & 0 & 0 \\ 0 & 0 & 0 & 0 & \ldots & 0 & 0 & G & 0 & 0 & 0 & 0 & 0 & \ldots & 0 & 0 & E & F \\ 0 & 0 & 0 & 0 & \ldots & 0 & 0 & G & 0 & 0 & 0 & 0 & 0 & \ldots & 0 & 0 & E & F \\ 0 & 0 & 0 & 0 & \ldots & 0 & 0 & G & F & E & 0 & 0 & 0 & \ldots & 0 & 0 & 0 & 0 \\ 0 & 0 & 0 & 0 & \ldots & 0 & 0 & G & F & E & 0 & 0 & 0 & \ldots & 0 & 0 & 0 & 0\end{array}\right)$

\subsection{The synthetic $\bar{X}$ chart with variable sample size (SVSS $\bar{X}$ chart)}

The synthetic chart combined with the VSS $\bar{X}$ chart signals when a sample point falls in the action region or two points, not far from each other, fall in the warning region-two. As the two points might be on opposite sides of the centerline, the event " $\underline{1}$ " is not necessary anymore, and the event " 1 " has now the following meaning $w_{c}<\left|Z_{i}\left(n_{i}\right)\right|<k$. The transition matrix (5) reduces to matrix (8).

\begin{tabular}{|c|c|c|c|c|c|c|c|c|c|c|c|c|c|}
\hline & $\begin{array}{c}0 \\
0 \\
\ldots \\
0 \\
0_{w}\end{array}$ & $\begin{array}{c}0 \\
0 \\
\ldots \\
0 \\
0_{c}\end{array}$ & $\begin{array}{c}0 \\
0 \\
\ldots \\
0 \\
1\end{array}$ & $\begin{array}{c}0 \\
0 \\
\ldots \\
1 \\
0_{\mathrm{c}}\end{array}$ & $\begin{array}{c}0 \\
0 \\
\ldots \\
1 \\
0_{w}\end{array}$ & $\begin{array}{l}0 \\
\ldots \\
1 \\
0 \\
0_{\mathrm{c}} \\
\end{array}$ & $\begin{array}{c}0 \\
\ldots \\
1 \\
0 \\
0_{w}\end{array}$ & $\cdots$ & $\begin{array}{l}0 \\
1 \\
\ldots \\
0 \\
0_{\mathrm{c}}\end{array}$ & $\begin{array}{l}0 \\
1 \\
\ldots \\
0 \\
0_{w}\end{array}$ & $\begin{array}{c}1 \\
0 \\
\ldots \\
0 \\
0_{\mathrm{c}}\end{array}$ & $\begin{array}{c}1 \\
0 \\
\ldots \\
0 \\
0_{w}\end{array}$ & Signal \\
\hline $00 \ldots 00_{c}$ & $\mathrm{~B}^{-}$ & $\mathbf{A}^{-}$ & $\mathrm{C}^{-}$ & 0 & 0 & 0 & 0 & $\ldots$ & 0 & 0 & 0 & 0 & $\mathbf{D}^{-}$ \\
\hline $00 \ldots 00_{w}$ & $\mathbf{B}^{+}$ & $\mathbf{A}^{+}$ & $\mathrm{C}^{+}$ & 0 & 0 & 0 & 0 & $\cdots$ & 0 & 0 & 0 & 0 & $\mathrm{D}^{+}$ \\
\hline $00 \ldots 01$ & 0 & 0 & 0 & $\mathbf{A}^{+}$ & $\mathbf{B}^{+}$ & 0 & 0 & $\cdots$ & 0 & 0 & 0 & 0 & $\mathbf{D}^{+}$ \\
\hline $00 \ldots 10_{c}$ & 0 & 0 & 0 & 0 & 0 & $\mathbf{A}^{-}$ & $\mathrm{B}^{-}$ & $\ldots$ & 0 & 0 & 0 & 0 & $\mathbf{D}^{-}$ \\
\hline $00 \ldots 10_{\mathrm{w}}$ & 0 & 0 & 0 & 0 & 0 & $\mathbf{A}^{+}$ & $\mathrm{B}^{+}$ & & 0 & 0 & 0 & 0 & $\mathbf{D}^{+}$ \\
\hline$\cdots$ & $\cdots$ & $\cdots$ & $\ldots$ & $\ldots$ & $\ldots$ & $\ldots$ & $\ldots$ & $\cdots$ & $\ldots$ & $\ldots$ & $\ldots$ & $\ldots$ & $\ldots$ \\
\hline $01 \ldots 00_{\mathrm{c}}$ & 0 & 0 & 0 & 0 & 0 & 0 & 0 & $\ldots$ & 0 & 0 & $\mathbf{A}^{-}$ & $\mathrm{B}^{-}$ & $\mathbf{D}^{-}$ \\
\hline $01 \ldots 00_{\mathrm{w}}$ & 0 & 0 & 0 & 0 & 0 & 0 & 0 & $\ldots$ & 0 & 0 & $\mathbf{A}^{+}$ & $\mathbf{B}^{+}$ & $\mathrm{D}^{+}$ \\
\hline $10 \ldots 00_{\mathrm{c}}$ & $\mathbf{B}^{-}$ & $\mathbf{A}^{-}$ & 0 & 0 & 0 & 0 & 0 & $\ldots$ & 0 & 0 & 0 & 0 & $\mathbf{D}^{-}$ \\
\hline $10 \ldots 00_{w}$ & $\mathbf{B}^{+}$ & $\mathbf{A}^{+}$ & 0 & 0 & 0 & 0 & 0 & $\ldots$ & 0 & 0 & 0 & 0 & $\mathrm{D}^{+}$ \\
\hline
\end{tabular}


Table 3

The $4 L$ elements of $\mathbf{S}_{C}$ and $\mathbf{N}$.

\begin{tabular}{|c|c|c|c|}
\hline$i$ & $S_{C}(i)$ & $i=1, \ldots, L-1$ & $N(i)$ \\
\hline $1,4 L$ & $F \times(E+F)^{L-2}$ & $2 L-2 i$ & $n_{1}$ \\
\hline $2,4 L-1$ & $E \times(E+F)^{L-2}$ & $2 L+2+2 i$ & \\
\hline $3,4 L-2$ & $F \times(E+F)^{L-3}$ & & \\
\hline $4,4 L-3$ & $E \times(E+F)^{L-3}$ & $2 L-1-2 i$ & $n_{2}$ \\
\hline$\ldots$ & $\ldots$ & $2 L+3+2 i$ & \\
\hline $2 L-5,2 L+6$ & $F \times(E+F)$ & & \\
\hline $2 L-4,2 L+5$ & $E \times(E+F)$ & $i$ & $N(i)$ \\
\hline $2 L-3,2 L+4$ & $F$ & $2 L+1$ & $n_{1}$ \\
\hline $2 L-2,2 L+3$ & $E$ & & \\
\hline $2 L-1,2 L+2$ & 1 & $2 L-1$ & \\
\hline $2 L$ & $2 F(E+F)^{L-1}+B_{0}(E+F)^{L} / C_{0}$ & $2 L$ & $n_{2}$ \\
\hline $2 L+1$ & $2 E(E+F)^{L-1}+A_{0}(E+F)^{L} / C_{0}$ & $2 L+2$ & \\
\hline
\end{tabular}

Table 4

Effect of $L$ on the SSARL of the SSVSS $\bar{X}$ chart $\left(k=3.5, w=0.672 n_{1}=2, n_{2}=6, \bar{n}=4\right)$.

\begin{tabular}{|c|c|c|c|c|c|c|}
\hline$L$ & 1 & 2 & 3 & 4 & 5 & 6 \\
\hline$w_{c}$ & 1.822 & 1.965 & 2.044 & 2.098 & 2.138 & 2.171 \\
\hline \multicolumn{7}{|l|}{$\delta$} \\
\hline 0 & 370.4 & 370.4 & 370.4 & 370.4 & 370.4 & 370.4 \\
\hline 0.2 & 132.5 & 132.7 & 131.8 & 131.0 & 130.3 & 129.9 \\
\hline 0.4 & 33.1 & 32.5 & 32.1 & 32.0 & 31.7 & 31.9 \\
\hline 0.6 & 11.1 & 10.7 & 10.7 & 10.7 & 10.8 & 10.9 \\
\hline 0.8 & 5.2 & 5.0 & 5.1 & 5.1 & 5.2 & 5.3 \\
\hline 1 & 3.2 & 3.2 & 3.2 & 3.2 & 3.3 & 3.3 \\
\hline 1.2 & 2.4 & 2.4 & 2.4 & 2.4 & 2.4 & 2.5 \\
\hline 1.4 & 1.9 & 2.0 & 2.0 & 2.0 & 2.0 & 2.0 \\
\hline 1.6 & 1.7 & 1.7 & 1.7 & 1.7 & 1.7 & 1.7 \\
\hline 1.8 & 1.5 & 1.5 & 1.5 & 1.5 & 1.5 & 1.5 \\
\hline 2 & 1.4 & 1.4 & 1.4 & 1.4 & 1.4 & 1.4 \\
\hline$E Q L$ & 21.63 & 21.50 & 21.49 & 21.54 & 21.60 & 21.66 \\
\hline
\end{tabular}

Table 5

Effect of $k$ on the SSARL of the SSVSS $\bar{X}$ chart $\left(L=3, w=0.672, n_{1}=2, n_{2}=6, \bar{n}=4\right)$.

\begin{tabular}{|c|c|c|c|c|c|c|c|}
\hline$k$ & 3.1 & 3.2 & 3.3 & 3.4 & 3.5 & 4 & 8 \\
\hline$w_{c}$ & 2.239 & 2.141 & 2.092 & 2.062 & 2.044 & 2.012 & 2.007 \\
\hline \multicolumn{8}{|l|}{$\delta$} \\
\hline 0 & 370.4 & 370.4 & 370.4 & 370.4 & 370.4 & 370.4 & 370.4 \\
\hline 0.2 & 158.0 & 144.8 & 138.0 & 134.2 & 131.8 & 128.9 & 128.9 \\
\hline 0.4 & 40.5 & 35.9 & 33.9 & 32.8 & 32.1 & 31.5 & 31.5 \\
\hline 0.6 & 12.7 & 11.5 & 11.0 & 10.8 & 10.7 & 10.7 & 10.7 \\
\hline 0.8 & 5.5 & 5.2 & 5.1 & 5.1 & 5.1 & 5.2 & 5.2 \\
\hline 1 & 3.2 & 3.2 & 3.2 & 3.2 & 3.2 & 3.3 & 3.3 \\
\hline 1.2 & 2.3 & 2.3 & 2.3 & 2.4 & 2.4 & 2.5 & 2.5 \\
\hline 1.4 & 1.9 & 1.9 & 1.9 & 1.9 & 2.0 & 2.1 & 2.1 \\
\hline 1.6 & 1.6 & 1.6 & 1.6 & 1.7 & 1.7 & 1.9 & 1.9 \\
\hline 1.8 & 1.4 & 1.5 & 1.5 & 1.5 & 1.5 & 1.7 & 1.7 \\
\hline 2 & 1.3 & 1.4 & 1.4 & 1.4 & 1.4 & 1.5 & 1.5 \\
\hline$E Q L$ & 22.53 & 21.71 & 21.45 & 21.42 & 21.49 & 22.41 & 25.03 \\
\hline
\end{tabular}

Again, the $Z\left(n_{i}\right)=\left(\bar{X}-\mu_{0}\right) \sqrt{n_{i}} / \sigma$ values, shortly $Z$ values, of the last $L$ samples define the transient states; " $0_{c}$ " means $|Z|<w$, " $0_{w}$ " means $w<|Z|<w_{c}$, and " 1 " means $w_{c}<|Z|<k$. With large samples, the events " $0_{w}$ ", " $0_{c}$ " and " 1 " occur with probabilities, $B^{+}, A^{+}$and $C^{+}$; with small samples, they occur with probabilities $B^{-}, A^{-}$and $C^{-}$. All these probabilities are the ones given in Table 2 , except that $C^{+}=\operatorname{Pr}\left[w_{c}<\left|Z\left(n_{2}\right)+\delta \sqrt{n_{2}}\right|<k\right]$ and $C^{-}=\operatorname{Pr}\left[w_{c}<\right.$ $\left.\left|Z\left(n_{1}\right)+\delta \sqrt{n_{1}}\right|<k\right]$. The remaining calculations to determine the steady-state ARLs of the synthetic $\bar{X}$ with variable sample size are similar to those presented for the side-sensitive version of the synthetic VSS $\bar{X}$ chart.

\subsection{The performance and the design of the VSS synthetic charts}

Table 4 presents the SSARLs and the EQL of the SSVSS $\bar{X}$ chart for $A R L_{0}=370.4, k=3.5, \delta_{\max }=2.0$ and $L$ varying from 1 to 6 . The $A R L_{0}$ is the in-control value of the steady state ARL. In Table 4, each row corresponds to a specified mean shift and, in each row, the numbers in bold correspond the lowest SSARLs. The parameter $L$ enhances the detection of small shifts ( $\delta \cong 0.5$ ). The $E Q L$ decreases as $L$ increases from 1 to 3 ; after that, the $E Q L$ increases with $L$. The lowest $E Q L$ is reached with $L=3(E Q L=21.49)$.

Table 5 presents the SSARLs and the EQL of the SSVSS- $\bar{X}$ chart for $A R L_{0}=370.4, L=5, \delta_{\max }=2.0$ and different values of $k$. In Table 5, each row corresponds to a specified mean shift and, in each row, the numbers in bold correspond the lowest SSARLs. A larger value of $k$ enhances the detection of small shifts. The EQL decreases as the value of $k$ increases from 3.1 to 3.4; after that, the $E Q L$ increases. The lowest $E Q L$ is reached with $k=3.4(E Q L=21.42)$.

Tables 6 and 7 compare the SSARL and the SSANOS of the standard VSS chart, that is, the one proposed by Costa (1994), with the SVSS $\bar{X}$ and the SSVSS $\bar{X}$ charts. These Tables also contain the $E Q L$ values for $\delta_{\max }=2.0$. In Tables 6 and 7, the lowest SSARLs, 
Table 6

The steady state $A R L$ and $A N O S$ of the VSS charts $\left(L=3, A R L_{0}=370.4\right)$.

\begin{tabular}{|c|c|c|c|c|c|c|c|c|c|c|c|c|c|c|}
\hline \multirow{3}{*}{$\begin{array}{l}\mathbf{w}_{c} \\
w\end{array}$} & \multicolumn{7}{|c|}{$\bar{n}=2 n_{1}=1 n_{2}=3$ ANOS $_{0}=740.8$} & \multicolumn{7}{|c|}{$\bar{n}=4 n_{1}=2 n_{2}=6$ ANOS $_{0}=1481.6$} \\
\hline & & & & 2.179 & & 2.044 & & & & & 2.179 & & 2.044 & \\
\hline & & 0.672 & & 0.672 & & 0.672 & & & 0.672 & & 0.672 & & 0.672 & \\
\hline$k$ & 3.0 & 3.0 & & 3.5 & & 3.5 & & 3.0 & 3.0 & & 3.5 & & 3.5 & \\
\hline & $\bar{X}$ & VSS & & SVSS & & SVSS & & $\bar{X}$ & VSS & & SVSS & & SVSS & \\
\hline$\delta$ & & $A R L$ & ANOS & $A R L$ & ANOS & $A R L$ & ANOS & & ARL & ANOS & ARL & ANOS & $A R L$ & ANOS \\
\hline 0.2 & 263 & 261 & 521 & 244 & 497 & 202 & 410 & 200 & 195 & 781 & 173 & 714 & 132 & 544 \\
\hline 0.4 & 131 & 122 & 244 & 98.7 & 210 & 71.6 & 152 & 71.6 & 61.0 & 243 & 43.8 & 196 & 32.1 & 143 \\
\hline 0.6 & 63.4 & 52.8 & 105 & 37.2 & 84 & 27.6 & 62 & 27.8 & 19.9 & 78 & 13.3 & 65 & 10.7 & 52 \\
\hline 0.8 & 32.4 & 23.8 & 47 & 15.9 & 38 & 12.6 & 30 & 12.4 & 7.9 & 31 & 5.8 & 29 & 5.1 & 26 \\
\hline 1 & 17.7 & 11.8 & 23 & 8.1 & 20 & 6.9 & 17 & 6.3 & 4.1 & 15 & 3.4 & 18 & 3.2 & 16 \\
\hline 1.2 & 10.4 & 6.6 & 12 & 5.0 & 13 & 4.4 & 11 & 3.6 & 2.6 & 9.4 & 2.5 & 12 & 2.4 & 12 \\
\hline 1.4 & 6.5 & 4.2 & 7.9 & 3.5 & 8.9 & 3.2 & 8.2 & 2.4 & 1.9 & 6.9 & 2.0 & 9.8 & 2.0 & 9.6 \\
\hline 1.6 & 4.3 & 3.0 & 5.4 & 2.7 & 6.9 & 2.6 & 6.5 & 1.7 & 1.6 & 5.6 & 1.7 & 8.2 & 1.7 & 8.1 \\
\hline 1.8 & 3.1 & 2.3 & 4.1 & 2.3 & 5.6 & 2.2 & 5.5 & 1.4 & 1.4 & 5.0 & 1.5 & 7.1 & 1.5 & 7.1 \\
\hline 2 & 2.3 & 1.9 & 3.4 & 2.0 & 4.8 & 1.9 & 4.7 & 1.2 & 1.3 & 4.7 & 1.4 & 6.5 & 1.4 & 6.5 \\
\hline$E Q L$ & 75.4 & 58.2 & & 46.8 & & 39.2 & & 33.6 & 27.1 & & 24.1 & & 21.5 & \\
\hline
\end{tabular}

Table 7

The steady state $A R L$ and ANOS of the VSS charts $\left(L=3, \mathrm{ARL}_{0}=700.0\right)$.

\begin{tabular}{|c|c|c|c|c|c|c|c|c|c|c|c|c|c|c|}
\hline \multirow{3}{*}{$\begin{array}{l}w_{c} \\
w\end{array}$} & \multicolumn{7}{|c|}{$\bar{n}=2 n_{1}=1 n_{2}=3$ ANOS $_{0}=1400.0$} & \multicolumn{7}{|c|}{$\bar{n}=4 n_{1}=2 n_{2}=6$ ANOS $_{0}=2800.0$} \\
\hline & & & & 2.345 & & 2.217 & & & & & 2.345 & & 2.217 & \\
\hline & & 0.674 & & 0.673 & & 0.674 & & & 0.673 & & 0.673 & & 0.674 & \\
\hline \multirow[t]{2}{*}{$k$} & 3.189 & 3.189 & & 3.5 & & 3.5 & & 3.189 & 3.189 & & 3.5 & & 3.5 & \\
\hline & $\bar{X}$ & VSS & & SVSS & & SVSS & & $\bar{X}$ & VSS & & SVSS & & SVSS & \\
\hline$\delta$ & & $A R L$ & ANOS & $A R L$ & ANOS & $A R L$ & ANOS & & $A R L$ & ANOS & $A R L$ & ANOS & $A R L$ & ANOS \\
\hline 0.2 & 479 & 474 & 949 & 441 & 897 & 367 & 746 & 356 & 346 & 1383 & 302 & 1246 & 233 & 961 \\
\hline 0.4 & 225 & 208 & 416 & 164 & 348 & 120 & 255 & 118 & 98.4 & 392 & 67.2 & 302 & 49.6 & 222 \\
\hline 0.6 & 104 & 84.3 & 168 & 56.2 & 128 & 41.8 & 95 & 42.8 & 29.2 & 116 & 17.9 & 88 & 14.3 & 70 \\
\hline 0.8 & 50.4 & 35.5 & 71 & 21.8 & 53 & 17.2 & 42 & 17.8 & 10.7 & 42 & 7.0 & 36 & 6.1 & 31 \\
\hline 1 & 26.3 & 16.5 & 32 & 10.3 & 26 & 8.6 & 22 & 8.5 & 5.0 & 19 & 3.8 & 20 & 3.5 & 18 \\
\hline 1.2 & 14.7 & 8.7 & 17 & 5.9 & 15 & 5.2 & 13 & 4.6 & 3.0 & 11 & 2.6 & 13 & 2.5 & 13 \\
\hline 1.4 & 8.8 & 5.2 & 9.9 & 3.9 & 10 & 3.6 & 9.3 & 2.9 & 2.1 & 7.6 & 2.1 & 10 & 2.0 & 10 \\
\hline 1.6 & 5.6 & 3.5 & 6.5 & 2.9 & 7.5 & 2.8 & 7.1 & 2.0 & 1.7 & 6.0 & 1.8 & 8.4 & 1.7 & 8.3 \\
\hline 1.8 & 3.8 & 2.6 & 4.7 & 2.4 & 6.0 & 2.3 & 5.8 & 1.5 & 1.5 & 5.2 & 1.6 & 7.2 & 1.6 & 7.2 \\
\hline 2 & 2.8 & 2.1 & 3.7 & 2.0 & 5.0 & 2.0 & 4.9 & 1.3 & 1.4 & 4.8 & 1.4 & 6.5 & 1.4 & 6.5 \\
\hline$E Q L$ & 114 & 85.1 & & 63.9 & & 52.9 & & 47.9 & 37.6 & & 30.4 & & 26.2 & \\
\hline
\end{tabular}

Table 8

VSS EWMA and SSVSS charts.

\begin{tabular}{llll}
\hline & $n_{1} / \bar{n}=0.6$ & $n_{2} / \bar{n}=4.0$ & $n_{1}=3, n_{2}=20, n_{\text {bar }}=20$ \\
& $H=0.372$ & $H=0.629$ & $w_{\mathrm{c}}=1.781$ \\
& $h=0.280$ & $h=0.430$ & $w=1.554$ \\
& $\lambda=0.044$ & $\lambda=0.099$ & $k=5.000$ \\
$\delta$ & VSS EWMA & VSS EWMA & SSVSS \\
\hline 0 & 370.4 & 370.4 & 370.4 \\
0.50 & 24.68 & 35.18 & 54.17 \\
0.75 & 14.50 & 14.50 & 20.85 \\
1.00 & 10.36 & 9.18 & 10.05 \\
1.50 & 6.68 & 5.51 & 4.32 \\
2 & 4.99 & 4.03 & 3.01 \\
$E Q L$ & 29.8 & 27.3 & 28.5 \\
\hline
\end{tabular}

ANOSs and EQLs are in bold. The SSVSS $\bar{X}$ chart always has smaller EQLs than the VSS and the SVSS $\bar{X}$ charts. However, the VSS chart requires less observations than the SSVSS chart to signal mean shifts larger than one standard deviation. The use of the SSVSS $\bar{X}$ chart is highly recommended when the rate of inspection and the false alarm risk are low; the highest $E Q L$ reductions are observed with $\bar{n}=2$ and $A R L_{0}=700.0$.

Table 8 compares the SSVSS $\bar{X}$ chart with the VSS EWMA chart proposed by Reynolds and Arnold (2001). They considered the EWMA control statistic

$Y_{k}=(1-\lambda) Y_{k-1}+\lambda Z_{k}$ where

$Z_{k}=\sqrt{\bar{n}} \frac{\bar{X}_{k}-\mu_{0}}{\sigma}$

The EWMA signals that the process mean has changed when $\left|Y_{k}\right| \geqslant H$, where $H$ is the control limit. With the VSS scheme, the next sample will be small if $\left|Y_{k}\right|<h$, or large if $h \leqslant\left|Y_{k}\right|<H$. The pair of design parameters $(H, \lambda)=(0.372,0.044)$ is the optimal one for detecting a shift $\delta=0.5$ while the pair of design parameters $(H, \lambda)$ $=(0.629,0.099)$ is the optimal one for detecting a shift $\delta=0.5$. The EWMA chart is very fast in signaling small mean shifts, but slower than the SSVSS chart in signaling larger disturbances. The EQLS in Table 8 were computed for $\delta_{\max }=2.0$.

Tables 9 and 10 deal with the design of the control charts, the SSVSS $\bar{X}$ charts in these Tables were designed to detect a specified mean shift with an in-control SSARL of 370.4 and an in-control rate of inspected items per sampling of 2 (Table 9) or 4 (Table 10). These Tables present two sets of SSVSS charts and in the last row the standard $\bar{X}$ chart. In the first set $n_{2}$ is fixed and slightly larger than $\bar{n}$; in Table $9, n_{2}$ is equal to 3 and, in Table $10, n_{2}$ is equal to 6 . In the second set, $n_{2}$ is free of restrictions. The first set deals with the cases where operational constraints do not allow working with large samples. Regarding to the first set of charts, smaller disturbances require larger $L$ values for the quickest detection of the specified mean shift. With respect to the second set, the fastest 
Table 9

The optimal design parameters for the SSVSS $\bar{X}$ chart, $\bar{n}=2, A R L_{0}=370.4$.

\begin{tabular}{|c|c|c|c|c|c|c|c|c|c|c|c|c|c|}
\hline & & & & & & & $\delta$ & & & & & & $E Q L$ \\
\hline$L$ & $n_{1}$ & $n_{2}$ & $w$ & $w_{c}$ & $k$ & $\delta^{*}$ & 0.25 & 0.50 & 0.75 & 1.00 & 1.25 & 1.50 & \\
\hline 7 & 1 & 3 & 0.67 & 2.17 & 4.2 & 0.5 & 150 & 41.9 & 15.0 & 7.23 & 4.44 & 3.22 & 49.7 \\
\hline 4 & & & & 2.07 & 3.8 & 0.75 & 153 & 42.4 & 14.8 & 6.93 & 4.18 & 3.01 & 48.7 \\
\hline 3 & & & & 2.03 & 3.6 & 1.00 & 155 & 43.2 & 15.0 & 6.87 & 4.08 & 2.91 & 48.7 \\
\hline 2 & & & & 2.02 & 3.3 & 1.50 & 164 & 46.4 & 15.8 & 7.01 & 4.02 & 2.80 & 50.3 \\
\hline 1 & 1 & 14 & 1.75 & 1.78 & 5.0 & 0.5 & 79.7 & 18.7 & 8.12 & 5.26 & 4.04 & 3.32 & 33.3 \\
\hline 1 & & 12 & 1.68 & 1.80 & 3.7 & 0.75 & 88.5 & 19.9 & 7.97 & 4.93 & 3.75 & 3.09 & 32.7 \\
\hline 1 & & 8 & 1.46 & 1.88 & 3.3 & 1.00 & 119 & 26.0 & 8.61 & 4.57 & 3.27 & 2.67 & 34.5 \\
\hline 1 & & 6 & 1.28 & 1.93 & 3.2 & 1.25 & 141 & 32.8 & 10.1 & 4.80 & 3.18 & 2.51 & 38.1 \\
\hline 1 & & 5 & 1.15 & 2.04 & 3.1 & 1.50 & 165 & 41.5 & 12.3 & 5.35 & 3.28 & 2.48 & 43.7 \\
\hline $\bar{X}$ & 2 & 2 & & & 3 & & 223 & 90.1 & 37.6 & 17.2 & 8.68 & 4.77 & \\
\hline
\end{tabular}

Table 10

The optimal design parameters for the SSVSS $\bar{X}$ chart, $\bar{n}=4, A R L_{0}=370.4$.

\begin{tabular}{|c|c|c|c|c|c|c|c|c|c|c|c|c|c|}
\hline & & & & & & & $\delta$ & & & & & & $E Q L$ \\
\hline$L$ & $n_{1}$ & $n_{2}$ & $w$ & $w_{c}$ & $k$ & $\delta^{*}$ & 0.25 & 0.50 & 0.75 & 1.00 & 1.25 & 1.50 & \\
\hline 5 & 1 & 6 & 0.53 & 2.11 & 4.0 & 0.5 & 88.2 & 17.3 & 6.09 & 3.44 & 2.50 & 2.04 & 25.2 \\
\hline 3 & 1 & & 0.53 & 2.04 & 3.5 & 0.75 & 91.6 & 17.6 & 5.90 & 3.21 & 2.29 & 1.85 & 24.4 \\
\hline 2 & 2 & & 0.67 & 2.02 & 3.3 & 1.00 & 96.4 & 18.6 & 5.99 & 3.12 & 2.17 & 1.75 & 24.5 \\
\hline 1 & 3 & & 0.96 & 2.04 & 3.1 & 1.50 & 113 & 22.7 & 6.82 & 3.24 & 2.12 & 1.66 & 26.9 \\
\hline 1 & 1 & 24 & 1.50 & 1.82 & 3.5 & 0.5 & 45.8 & 8.83 & 4.95 & 3.79 & 3.13 & 2.70 & 22.6 \\
\hline 1 & 1 & 14 & 1.19 & 1.93 & 3.2 & 0.75 & 69.9 & 10.5 & 4.24 & 2.97 & 2.46 & 2.18 & 22.1 \\
\hline 1 & 1 & 12 & 1.28 & 2.04 & 3.1 & 1.00 & 87.1 & 13.1 & 4.33 & 2.68 & 2.12 & 1.84 & 21.3 \\
\hline 1 & 1 & 10 & 1.46 & 2.04 & 3.1 & 1.25 & 96.0 & 16.3 & 4.95 & 2.73 & 2.02 & 1.70 & 22.6 \\
\hline 1 & 3 & 6 & 0.96 & 2.04 & 3.1 & 1.50 & 113 & 22.7 & 6.82 & 3.24 & 2.12 & 1.66 & 26.9 \\
\hline $\bar{X}$ & 4 & 4 & & & 3.0 & & 155 & 43.4 & 14.5 & 5.80 & 2.74 & 1.50 & \\
\hline
\end{tabular}

Table 11

The sample observations of the illustrative example.

\begin{tabular}{|c|c|c|c|c|c|c|c|c|}
\hline Sample number & Sample size & Sample & ations & & $\bar{X}$ value & $Z$ value & Sample classification & Symbol \\
\hline 1 & Small & 125.18 & & & 125.18 & -1.41 & Conforming & $\triangle$ \\
\hline 2 & Large & 129.29 & 127.76 & 128.07 & 128.37 & 0.32 & Conforming & $\mathrm{O}$ \\
\hline 3 & Small & 127.72 & & & 127.72 & -0.14 & Conforming & $\triangle$ \\
\hline 4 & Small & 130.72 & & & 130.72 & 1.36 & Conforming & $\triangle$ \\
\hline 5 & Large & 124.32 & 126.60 & 124.32 & 125.08 & -2.53 & Nonconforming & $\bullet$ \\
\hline 6 & Large & 126.56 & 129.68 & 127.94 & 128.06 & 0.05 & Conforming & O \\
\hline 7 & Small & 133.68 & & & 133.68 & -0.33 & Conforming & $\triangle$ \\
\hline 8 & Small & 162.08 & & & 162.08 & 2.84 & Nonconforming & $\Delta$ \\
\hline 9 & Large & 132.13 & 128.92 & 127.66 & 129.57 & 1.36 & Conforming & $\overline{0}$ \\
\hline 10 & Large & 129.46 & 130.49 & 133.92 & 131.29 & 2.85 & Nonconforming & $\bullet$ \\
\hline
\end{tabular}

detection of the specified mean shifts is always with $L=1$. The two sets of SSVSS charts present the values of their parameters: $L, n_{1}$, $n_{2}, w, w_{c}$ and $k$; in each row, they are the optimal values for the fastest detection of a specified shift $\delta=\delta^{*}$. The lowest SSARLs are in bold. Considering the two sets of SSVSS charts, the optimal $k$ value always decreases as the specified mean shift increases.

\section{An example illustrating the use of the SSVSS $\bar{X}$ chart}

A textile company controls the tensile strength of fibers used in manufacturing cloths, by taking samples of size two from the process and plotting the sample means on a $\bar{X}$ chart with control limits of three standard deviations. The target value $\left(\mu_{0}\right)$ of the tensile strength is 128 psi. Historical studies have shown that the process standard deviation $(\sigma)$ is 2 psi; see Mahadik and Shirke (2009).

The quality department is considering the joint use of the VSS scheme with the side-sensitive synthetic rule to reduce the SSARL, that is, the delay with which the $\bar{X}$ signals moderate mean shifts ( $\mu \approx \mu_{0} \pm \delta \sigma=130 \mathrm{psi}$, that is, $\delta=1.00$ ). The engineering and administrative requirements have specified three units as the maximum sample size. Table 9 shows that the matched SSVSS $\bar{X}$ chart with $\left(L, n_{1}, n_{2}, k\right)=(3,1,3,3.6)$ reduces the delay by more than $60 \%$, from 17.2 to 6.87 .

With the SSVSS chart in use, the observations of ten samples, including their $\bar{X}$ values, $Z$ values, classifications and corresponding symbols were registered in Table 11. The points in Fig. 2 are the $Z$ values of these samples. The sample observations were simulated from a normal distribution with standard deviation equal to 2 and the mean equal to 128 (for the first seven samples) or 130 (for the last three samples).

The point position not only classifies the sample but also defines the size of the next sample. If the point falls in any of the two warning regions, the next sample will be large; otherwise, it will be small. The two warning regions serve to distinguish conforming and nonconforming samples. The conforming sample points are in the warning region-one (or in the central region), whereas the nonconforming sample points are in the warning region-two.

In Fig. 2, the size of the first sample was randomly chosen to be small and the $C R L$ was equated to one. As the first $Z$ value fell in the 


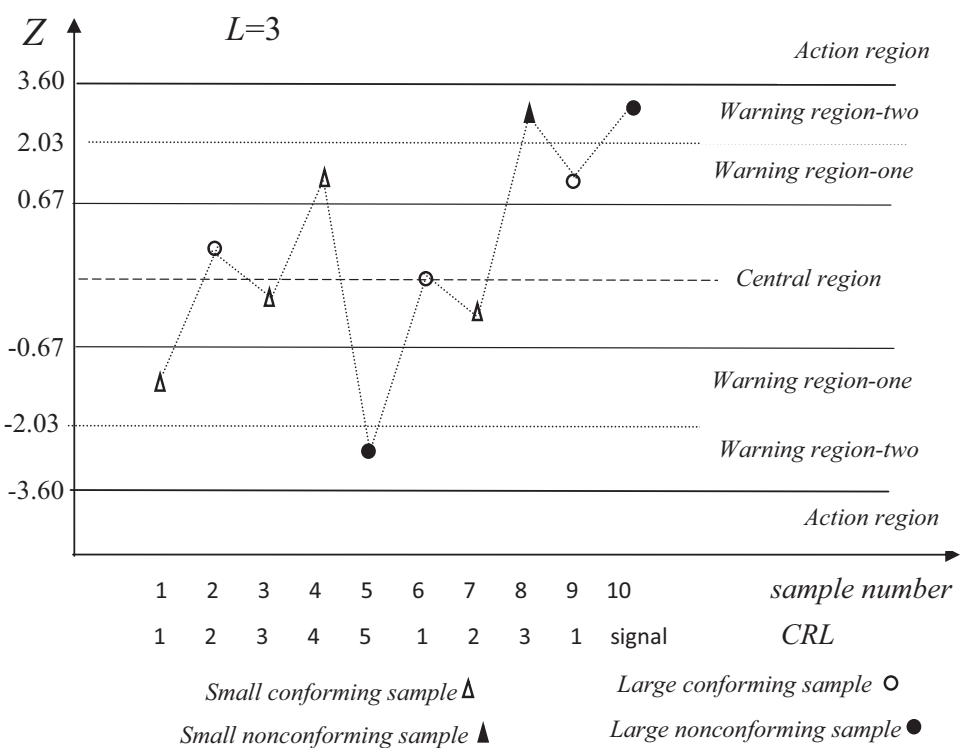

Fig. 2. The SSVSS $\bar{X}$ chart - an illustrative example.

lower-warning region-one, the second sample size was chosen to be large. The second $Z$ value fell in the central region, consequently, the third sample was chosen to be small and the CRL increased by one unit $(C R L=2)$. The third and fourth $Z$ values fell, respectively, in the central region and in the upper warning region-one, consequently the $C R L$ increased by two units $(C R L=4)$. Sample five is a nonconforming sample because its $Z$ value fell in the lower warning region-two. After the occurrence of a nonconforming sample, the $C R L$ is compared with $L$. The $C R L(=5)$ was observed to be larger than $L(=3)$; consequently, the $C R L$ returned to zero. The second point to fall in the warning region-two was the one corresponding to sample eight, but it fell on the opposite side, that is, in the upper warning region-two; this time the $C R L$ returned to zero due to the fact that the proposed chart is side-sensitive. The third point to fall in the warning region-two was the one corresponding to sample ten; as it fell on the same side of the second one, that is, in the upper warning region-two, the $C R L$ was again compared with $L$. Now the $C R L(=2)$ is smaller than $L(=3)$; consequently, the SSVSS $\bar{X}$ chart triggered a signal.

\section{Conclusions}

In this article, we investigated the performance of the side-sensitive synthetic chart combined with a VSS $\bar{X}$ chart. The side-sensitive feature always improves the synthetic chart's performance. This is explained by the fact that the occurrence of signals given by a second point beyond the warning limit - not far from the first one but plotted on the opposite side of the center line - reduces as the process mean shifts. This fact is also observed with the VSS scheme, that is, the SSVSS $\bar{X}$ chart always outperforms the SVSS $\bar{X}$ chart. As expected, the VSS EWMA chart signals small mean shifts faster than the SSVSS $\bar{X}$ chart. The SSVSS $\bar{X}$ chart is highly recommended to detect process mean shifts when the aim is to work with low risks of false alarms and very low rates of inspected items.

\section{Acknowledgements}

The authors wish to express their sincere thanks to the Referees and the Editor for their precious time reviewing our paper. They really helped us to improve our paper. This work was supported by CNPq - National Council for Scientific and Technological Development (Grant Nos. 306189/2011-9 and 301739/2010-2).

\section{References}

Acosta-Mejia, C. (2007). Two sets of runs rules for the chart. Quality Engineering, 19, 129-136.

Antzoulakos, D. L., \& Rakitzis, A. C. (2008). The revised m-of-k runs rule. Quality Engineering, 20, 75-81.

Bajirao, A. S., \& Parasharam, G. M. (2015). Modified synthetic control chart for onestep Markov-dependent processes. Communications in Statistics-Theory and Methods, 44, 942-952.

Castagliola, P., Zhang, Y., Costa, A., \& Maravelakis, P. (2012). The variable sample size $\bar{X}$ chart with estimated parameters. Quality and Reliability Engineering International, 28, 687-699.

Champ, C. W., \& Woodall, W. H. (1987). Exact results for Shewhart control charts with supplementary runs rules. Technometrics, 29, 393-399.

Chew, X. Y., Khoo, M. B. C., Teh, S. V., \& Castagliola, P. (2015). Variable sampling interval run sum $\bar{X}$ control chart. Computer and Industrial Engineering, 90, 25-38.

Chong, Z. L., Khoo, M. B. C., \& Castagliola, P. (2014). Synthetic double sampling np chart for attributes. Computer and Industrial Engineering, 75, 157-169.

Costa, A. F. B. (1994). $\bar{X}$ control charts with variable sample size. Journal of Quality Technology, 26, 155-163.

Costa, A. F. B. (1997). $\bar{X}$ charts with variable sample size and sampling intervals. Journal of Quality Technology, 29, 197-204.

Costa, A. F. B. (1998). Joint $\bar{X}$ and $R$ charts with variable parameters. IIE Transactions, 30, 505-514.

Costa, A. F. B. (1999a). Joint $\bar{X}$ and $R$ charts with variable sample sizes and sampling intervals. Journal of Quality Technology, 31, 387-397.

Costa, A. F. B. (1999b). $\bar{X}$ charts with variable parameters. Journal of Quality Technology, 31, 408-416.

Costa, A. F. B., De Magalhaes, M. S., \& Epprecht, E. (2009). Monitoring the process mean and variance using a synthetic control chart with two-stage testing. International Journal of Production Research, 18, 5067-5086.

Costa, A. F. B., \& Machado, M. A. G. (2011). Variable parameter and double sampling $X$ charts in the presence of correlation: The Markov chain approach. International Journal of Production Economics, 130, 224-229.

Costa, A. F. B., \& Machado, M. A. G. (2015). The steady-state behavior of the synthetic and side-sensitive synthetic double sampling $\bar{X}$ charts. Quality and Reliability Engineering International, 31, 297-303.

Davis, R. B., \& Woodall, W. H. (2002). Evaluating and improving the synthetic control chart. Journal of Quality Technology, 34, 200-208.

Guo, B., Wang, B. X., \& Cheng, Y. (2015). Optimal design of a synthetic chart for monitoring process dispersion with unknown in-control variance. Computer and Industrial Engineering, 88, 78-87.

Haridy, S., Wu, Z., Khoo, M. B. C., \& Yu, F. J. (2012). A combined synthetic and np scheme for detecting increases in fraction nonconforming. Computer and Industrial Engineering, 62, 979-988.

Haridy, S., Wu, Z., Abhary, K., Castagliola, P., \& Shamsuzzaman, M. (2014). Development of a multivariate synthetic-np chart. Journal of Statistical Computation and Simulation, 84, 1884-1903. 
Khoo, M. B. C., \& Ariffin, K. N. (2006). Two improved runs rules for the Shewhart control chart. Quality Engineering, 18, 1-6.

Khoo, M. B. C., Lee, H. C., Wu, Z., Chen, C. H., \& Castagliola, P. (2011). A synthetic double sampling chart for the process mean. IIE Transactions, 43, 23-38.

Khoo, M. B. C., Wu, Z., Castagliola, P., \& Lee, H. C. (2013). A multivariate synthetic double sampling $T^{2}$ control chart. Computer and Industrial Engineering, 64, 179-189.

Khoo, M. B. C., Tan, E. K., Chong, Z. L., \& Haridy, S. (2015). Side-sensitive group runs double sampling (SSGRDS) chart for detecting mean shifts. International Journal of Production Research, 53, 4735-4753.

Lee, P. H. (2013). Joint statistical design of $\bar{X}$ and $s$ charts with combined double sampling and variable sampling interval. European Journal of Operational Research, 225, 285-297.

Lee, M. H., Khoo, M. B. C., \& Xie, M. (2014). An optimal control procedure based on multivariate synthetic cumulative sum. Quality and Reliability Engineering International, 30, 1049-1058.

Lee, M. H., \& Khoo, M. B. C. (2015). Multivariate synthetic $|S|$ control chart with variable sampling interval. Communications in Statistics-Simulation and Computation, 44, 924-942.

Lim, S. L., Khoo, M. B. C., Teoh, W. L., \& Xie, M. (2015). Side-sensitive group runs double sampling (SSGRDS) chart for detecting mean shifts. International Journal of Production Economics, 166, 20-35.

Machado, M. A. G., Costa, A. F. B., \& Rahim, A. (2009). The synthetic control chart based on two sample variances for monitoring the covariance matrix. Quality and Reliability Engineering International, 25, 595-606.

Machado, M. A. G., \& Costa, A. F. B. (2014a). Some comments regarding the synthetic $\bar{X}$ chart. Communications in Statistics-Theory and Methods, 43, 2897-2906.

Machado, M. A. G., \& Costa, A. F. B. (2014b). A side-sensitive synthetic chart combined with an $\bar{X}$ chart. International Journal of Production Research, 52, 3404-3416.
Mahadik, S. B., \& Shirke, D. T. (2009). A special variable sample size and sampling interval $\bar{X}$ chart. Communications in Statistics-Theory and Methods, 38, 1284-1299.

Noorossana, R., Shekary, A., \& Deheshvar, A. (2015). Combined variable sample size sampling interval, and double sampling (CVSSIDS) adaptive control charts. Communications in Statistics-Theory and Methods, 44, 1255-1269.

Rajmanya, S. V., \& Ghute, V. B. (2014). A synthetic control chart for monitoring the process variability. Quality and Reliability Engineering International, 30, 1301-1309.

Reynolds, M. R., \& Arnold, J. C. (2001). EWMA control charts with variable sample sizes and with variable sampling intervals. IIE Transactions, 33, 511-530.

Western Electrical Company (1956). Statistical Quality Control Handbook, Western Electric Corporation, Indianapolis, IN.

Wu, Z., \& Spedding, T. A. (2000). A synthetic control chart for detecting small shifts in the process mean. Journal of Quality Technology, 32, 32-38.

Wu, Z., Yang, M., Jiang, W., \& Khoo, M. B. C. (2008). Optimization designs of the combined Shewhart-CUSUM control charts. Computational Statistics and Data Analysis, 53, 496-506.

Wu, Z. Ou, Y. Castagliola, P. \& Khoo, M. B. C. (2010). A combined synthetic \& X chart for monitoring the process mean. International Journal of Production Research, 48 $7423-7436$.

Yeong, W. C., Khoo, M. B. C., Wu, Z., \& Castagliola, P. (2012). Economically optimum design of a synthetic $\bar{X}$ chart. Quality and Reliability Engineering International, 28 , $725-741$.

Yeong, W. C., Khoo, M. B. C., Lee, M. H., \& Rahim, M. A. (2013). Economic and economic statistical designs of the synthetic $\bar{X}$ chart using loss functions. European Journal of Operational Research, 228, 571-581.

Zhang, Y., Castagliola, P., Wu, Z., \& Khoo, M. B. C. (2011). The synthetic $\bar{X}$ chart with estimated parameters. IIE Transactions, 43, 676-687. 